\title{
A walk round the edges of self tolerance
}

\author{
N A Mitchison
}

The immunologists of my generation were brought up on Sir Macfarlane Burnet's clonal selection theory. ${ }^{1}$ Anticipating Margaret Thatcher's view of society, we believed that all that matters in the immune system is the sum of the decisions taken by individual lymphocytes. Engagement of its receptor by antigen suffices to drive a lymphocyte to respond. For $T$ cells the only exception is the thymus, where engagement of the receptor by self antigen drives the cell into programmed cell death. This process of negative selection explains how the immune system avoids reacting against self. The two parts of the theory fitted neatly together, and nothing more seemed necessary. When cooperation between $T$ and $B$ cells came along, ${ }^{2}$ it too could be accommodated comfortably within the theory.

Later came Nils Jerne's idiotype network, with the proposal that lymphocytes use their receptors to recognise not only antigen but also the receptors of other lymphocytes. ${ }^{3}$ Opinion among immunologists divided, with heretics arguing that antigens work not by selection but by perturbing a network of interactive cells. The orthodox viewed the network merely as an inescapable but unhelpful consequence of clonal selection, which the immune system does its best to damp down by suppression.

\section{Challenge to clonal selection}

Over the past year or two Irun Cohen, from the Weizmann Institute in Israel, has been mounting a more radical attack on the clonal selection theory. ${ }^{4} \mathrm{He}$ draws partly on older information, such as the well established fact that many proteins prove unable to induce an immune response without the help of an adjuvant. This alone is sufficient to dispose of the idea that engaging a receptor with antigen is all that is required. Attempts to explain adjuvants simply as devices for maintaining the right local concentration of antigen have failed. Another of his arguments is the restricted range of autoimmune diseases. Burnet thought that these diseases resulted from mutations in receptor-encoding genes, so that each case should be unique. In reality the number of such diseases is quite small, perhaps less than 30 in all, with many subjects suffering from each one. Perhaps the most telling argument comes from recent studies on autoreactive $T$ cells in tissue culture. It has long been known that normal subjects contain occasional $T$ cells able to react against self $B$ cells and self macrophages, but it has not proved possible to identify just what antigens, if any, are involved. The position changed when well. defined proteins became available from studies on autoimmune diseases. All of the antigens identified as targets of autoimmunity so far tested prove able to stimulate $T$ cells from normal subjects. The list of proteins that are 'noisy' in this way includes myelin basic protein, heat shock proteins, acetylcholine receptor, and thyroid peroxidase. It probably also includes type II collagen, though a question mark hangs over earlier data because of contamination with the porcine pepsin used to solubilise the collagen. ${ }^{5}$ Nobody knows why $T$ cells of these types do not cause autoimmune disease in normal subjects. Some of these proteins may be too well hidden from cells of the immune system, but this seems unlikely to account for the whole phenomenon. It is tempting to believe that these $T$ cells are held normally under some kind of restraint, from which they escape in culture. The figure attempts to summarise this position.

Arguments of this sort have conjured up a certain rhetoric. Cohen refers to 'committee decisions', and Janeway to 'costimulation'. ${ }^{6}$ Grossman prefers 'contextual discrimination', a useful term because it avoids reference to any particular mechanism. We have to accept the fact that the challenge to clonal selection theory comes from a diffuse range of possibilities rather than a single, coherent idea. The problem is not the lack of possible mechanisms, but rather the plethora. The context within which a lymphocyte makes its decision is set by the totality of receptors and adhesion molecules that it carries on its

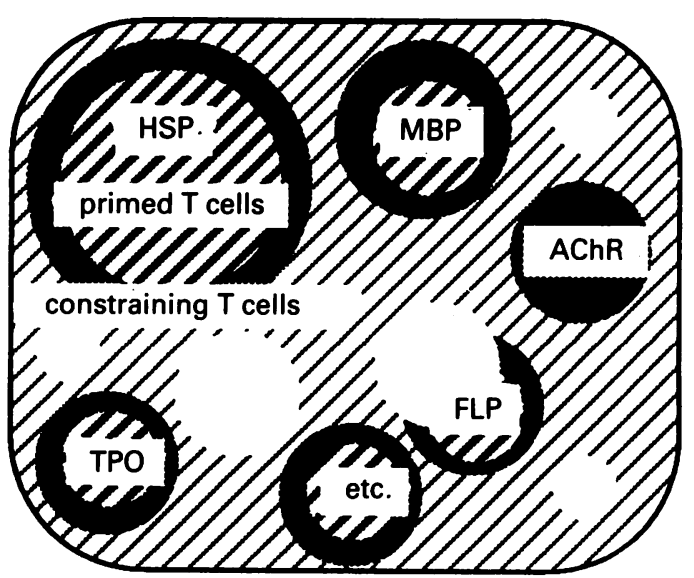

$A$ cartoon of the $T$ cell repertoire that combines Cohen's ideas with negative selection. HSP, MBP, AchR, TPO, and FLP are the noisy self proteins mentioned in the text. They prime $T$ cells, which in turn are contained by constraining $T$ cells. The repertoire also has holes, punched by negative selection in the thymus. The background repertoire has for the most part also been generated by postthymic positive selection, by environmental antigens. $H S P=$ heat shock proteins; $M B P=$ myelin basic protein, Ach $R=$ acetylcholine receptor; $T P O=$ thyroid peroxidase $F L P=F$ liver protein. 
surface, plus the cytokines and cell surface ligands to which it is exposed. Getting on for 100 proteins have been identified on the surface of human lymphocytes, so the choice is wide. Of course there are shades of opinion, often influenced by the personal experience of individual investigators. Some emphasise the importance of network interactions, others the cytokine cascade, and yet others the secondary signalling pathways served by particular adhesion-ligand pairs. There is no consensus, and insufficient data to base one on. The question is rather, what is the best experiment to do now?

Two pieces of my own experience draw me to this position. Guided by Hardy Cinader, then at the old Lister Institute, I had begun to study the antibody response of mice to foreign serum proteins such as albumin and immunoglobin. I confirmed Cinader's discovery that these proteins make excellent antigens when administered in adjuvant. My student David Dresser went on to discover that it was almost impossible to make a mouse respond to these soluble proteins without adjuvant, and that on the contrary they would become immunologically tolerant. ${ }^{8}$ Eventually, he found that the adjuvant did not even need to be mixed with the foreign protein in order to secure an antibody response but could be injected elsewhere in the body. This disposed of the local concentration argument, and told us quite clearly that individual lymphocytes must respond to two signals, one from the antigen and the other from the adjuvant.

My second experience springs from the first. After most of a lifetime spent in the study of immunological tolerance, my colleagues and I have at last managed to measure the concentration of a soluble protein that is needed to induce tolerance within the thymus. ${ }^{9}$ One takes the tiny thymus out of a mouse embryo and places it in culture, where it swells with multiplying and maturing $\mathrm{T}$ cells. One can add the protein into the culture, and find out whether it has induced tolerance in the maturing cells by transferring them back into adult mice and challenging them there with the protein plus adjuvant. The significant finding is that the concentration required to induce tolerance is just the same as that required to make tolerant a normal adult mouse, as measured previously by Dresser and myself. One can be quite sure that the thymus has nothing to do with Dresser's form of tolerance, as it makes no difference whether or not the adult mouse is thymectomised beforehand. We can also exclude the possibility that there is something abnormal about this kind of thymic function, as $T$ cells that mature within the thymus do normally become tolerant of at least one such soluble self protein from the liver. We conclude, contrary to the generally accepted view, that tolerance to these self proteins has nothing to do with any special susceptibility on the part of immature lymphocytes. Rather it seems that $T$ cells pass through some microenvironment that exists within both the thymus and the peripheral lymph nodes, where some signal (or 'costimulus') from a cytokine or an adhesion molecule that is needed for a positive response is lacking. Formally, one has to mention the alternative possibility of costimulation needed for tolerance induction rather than for the positive response. What my two experiences have in common is evidence of $\mathrm{T}$ cells modulating their response in accordance with signals received from other cells. That is why I respond enthusiastically to the idea of contextual discrimination.

So where does all this leave clonal selection? Cohen writes about the breaking of a paradigm. When he does so I reach, mentally, for my toaster, as that is where as a schoolboy I learned that a boaster should be put. It takes a generation to feel fully at home with a new concept, and I believe that it has taken us that long to come to terms with clonal selection. Now that we are quite confident about the ability of negative selection to account for most of tolerance of self, we can start probing the edges where natural selection, the great tinkerer, no doubt has some surprises for us. One is reminded of the probing of the edges of mendelian inheritance which took place in the 1940s. That too was separated from the rediscovery of Mendel's work by a generation, and was misinterpreted as a loss of confidence in mendelism.

\section{Response to self proximal immunogens} Most of the animal models of autoimmunity now in use can be traced back to the introduction of Freund's adjuvant in the $1940 \mathrm{~s}$, and the subsequent discovery of its ability to allow autoimmunisation with such tissues as spinal cord, peripheral nerve, uveal tract, and testis. Arthritis induced by type II collagen joined the list a little later. Other forms of insult could sometimes replace Freund's adjuvant, as in renal disease produced by in situ treatment of glomerular basement membrane with mercury ions. My point is that the older immunisation models (the purely genetical models are omitted from discussion as their underlying immunisation processes are so poorly understood) open only a fairly arbitrary window on self tolerance.

In trying to conceive how the window might be opened wider, I have been much influenced by the work of my colleague Susanne Schneider. She studies the response of mouse $\mathrm{T}$ cells to what is probably a single amino acid substituted variant of their own $F$ liver protein. ${ }^{9}$ The variant was chosen because it occurs naturally in certain mouse strains, and the system demonstrates alloimmunisation, leading to an exceptionally strong autoantibody response. So strong is this response that the protein, of unknown normal function, has long been recognised as immunologically 'noisy'. Susanne is discovering that it is also noisy as an autoantigen in the way mentioned above. Immunised mice contain $\mathrm{CD} 4 \mathrm{~T}$ cells which respond in culture to self-type $F$ liver protein, despite the fact that they make no detectable response to it in vivo. Because the protein has been cloned and sequenced she can now proceed with detailed epitope analysis. 
For this purpose she uses $\mathrm{T}$ cell hybridomas, a powerful technology for the mouse, which much needs transferring to man. This autoresponse to an alloprotein has obvious similarities to the well known autoimmunity that develops transiently in mice after immunisation with rat blood cells, but unfortunately that is not a system readily open to epitope analysis.

The need to search for other usable allovariations in protein structure has passed. These days one can obtain single amino acid substitutions at will by site specific mutagenesis, which could be used to generate self proximal antigens ad infinitum. This opens the way to a systematic probing of the borders of self tolerance.

In addition to this use in exploring the $T$ cell repertoire, self proximal antigens should have valuable immunogenetic features. As simple epitopes, they are likely to show strong major histocompatibility complex (MHC) associations. Experience, admittedly with only a tiny number of these antigens, suggests that not only are positive associations likely to be found but also negative ones. ${ }^{10}$ Such immunoinhibitory effects may well be analogous to the protection against rheumatoid arthritis displayed by HLA-DR4-Dw10 and DQ-Dw6. A deeper understanding of the mode of action of these protective genes is likely to lead to new therapeutic strategies: any mechanism which prevents a disease is likely also to help cure it.

\section{Collagen induced arthritis and the minimax family}

This experimental disease is admirably suited for such an investigation. The immunology is well understood, thanks largely to two vigorous groups of researchers in Uppsala. ${ }^{11}{ }^{12}$ Both B and $T$ cells are required, as judged by the need to use conformationally intact collagen on the one hand, and tight MHC associations on the other. The enormous gene for mouse type II collagen has just been cloned, in Turku, and cDNA of convenient length is available. ${ }^{13}$ There should be no difficulty in using this to produce engineered peptides, which could then be used to activate mouse $T$ cells. On their own these $T$ cells would not usually be expected to engender the disease, but they could be combined with $\mathrm{B}$ cells induced by conformationally intact collagen (probably bovine) in an adoptive transfer. This would conform to the standard experimental design, in which a single batch of activated B cells is used to assess the activity of several $T$ cell populations. ${ }^{14}$

More of us should work on the immunology of collagen. This fascinating family of proteins includes members which are widely distributed in the body and therefore induce a high degree of self tolerance. Others have a more restricted distribution, and therefore enjoy a measure of immunological privilege. Hiding away from the immune system is more complicated than one might imagine, as recent experiments with transgenes show that not only do location and concentration matter, but also access to professional antigen presenting cells. ${ }^{15}$ However it works in detail, privilege should lower the effective concentration of these collagens in the vicinity of $T$ cells undergoing negative selection. Studies on lysozyme transgenes show that different epitopes mediate negative selection at different concentrations. ${ }^{16}$ Accordingly we can expect the collagens to comprise a minimax family, in which some members establish self tolerance more completely than others, and in which certain epitopes will be fully tolerated on some members, though homologous structures on others will not.

\section{Purpose of animal models}

Up to a point the collagen arthritis model confuses two issues: the $T$ cell repertoire at the edge of self tolerance, and the ability of $\mathrm{T}$ cells from that region to induce disease. This does not seem to me much of an objection while we are still looking for clues to disease epitopes. More generally, the validity of the animal models for their human diseases has been widely questioned, but again I would argue that at our present stage that does not matter. The universes of the immunologically noisy self proteins and of the disease inducing ones, and the extent of their overlap, urgently need exploration. In that enterprise we need all the help that we can get from animal models.

1 Burnet M. The clonal selection theory of acquired immunity. Nashville, Tennessee: Vanderbilt University Press, 1959.

2 Claman H N, Chaperon E A, Triplett RF. Thymus-marrow cell combinations. Synergism in antibody production. Proc Soc Exp Biol Med 1966; 122: 1167.

3 Jerne $\mathrm{N} \mathrm{K}$. Towards a network theory of the immune system. Ann Immunol (Inst Pasteur) 1974; 125C: 373

4 Cohen I R, Young D B. Autoimmunity, microbial immunity and the immunological homunculus. Immunol Today 1991; 12: 105-10.

5 Lacour M, Rudolphi U, Schlesier M, Peter H H. Type II collagen-specific human $T$ cell lines established from healthy donors. Eur f Immunol 1990; 20: 931-4

6 Janeway C A. Approaching the asymptote? Evolution and revolution in immunology. Cold Spring Harb Symp Quant Biol 1989; 54: 1-14.

7 Grossman Z. Contextual discrimination of antigens by the immune system: towards a unifying hypothesis. In: Sercarz E, Berzofsky J, eds. Immunogenicity of protein antigens: repertoire and regulation. Vol II. Boca Raton: CRC antigens: repertoire and

8 Dresser D W. Tolerance induction as a model for cell differentiation. Br Med Bull 1976; 32: 147-51.

9 Robertson K, Schneider S, Simon K, Timms E, Mitchison $\mathrm{N} \mathrm{A}$. Tolerance of self induced in thymus organ culture. Eur f Immunol 1992; 22: 207-11.

10 Mitchison N A, Simon K. Dominant unresponsiveness controlled by $\mathrm{H}-2 \mathrm{Ab}$. A new pattern displayed by Thy1 antigen and $F$ liver antigen. Immunogenetics 1990; 32: $104-9$.

11 Holmdahl $R \quad M$, Andersson $M$, Goldschmidt $T$ J Gustafsson K, Jansson L, Mo J A. Type II collagen Gustafsson K, Jansson L, Mo J A. Type II collagen autoimmunity in animals and provocations lea

12 Klareskog L, Olsson T. Autoimmunity to collagen II and myelin basic protein: comparative studies in humans and rodents. Immunol Rev 1990; (No 118): 285-310.

13 Metsäranta $M$, Toman D, de Crombrugghe B, Vuorio E. Mouse type II collagen gene. 7 Biol Chem 1991; 266: 16862-9.

14 Lightstone E B, Marvel J, Mitchison N A. Memory in helper $T$ cells revealed in vivo by alloimmunizations in combination with Thyl antigen. Eur f Immunol 1992; 22: 115-22.

15 Zinkernagel R M, Cooper S, Chambers J, Lazzarini R A Hengartner H, Arnheiter H. Virus-induced autoantibody responses to a transgenic viral antigen. Nature $1990 ; 345$ 68-71

16 Cibotti R, Kanellopoulus J M, Canabiols J P, et al. Tolerance to a self-protein involves its immunodominan but not subdominant determinants. Proc Natl Acad S USA 1991; 89: 416-20. 